\title{
Scale corrections for faster evaluation of convergent Fresnel patterns
}

\author{
DAVID MAS*, JULIÁN ESPINOSA, JORGE PÉREZ and \\ CARLOS ILLUECA \\ Departamento Optica, Universidad de Alicante, PO Box 99, \\ 03080 Alicante, Spain
}

(Received 26 May 2005)

\begin{abstract}
Numerical calculation of Fresnel patterns through fast Fourier transforms usually requires an extremely large number of samples in order to fulfil the Nyquist sampling condition. In many applications, the cut-off frequency of the system is much below the limit fixed by our calculations. As a consequence of this, correct sampling may result in heavy processes that produce results of useless accuracy. Unfortunately, subsampling may introduce aliasing that may distort the final appearance of the diffracted pattern. In this paper, we present a simple method that permits subsampling the Fresnel pattern while maintaining the Nyquist condition, and thus preventing the appearance of aliasing effects in the calculation. Secondary effects of the subsamplings are rescaling the illuminating wavelength and the introduction of an effective low-pass filter. Some applications relative to the propagation of the light inside the human eye are also suggested in the text.
\end{abstract}

\section{Introduction}

Numerical calculation of optical transformations is a recurrent topic in optics [1-7]. Among these transformations we include Fourier transforms, fractional Fourier transforms, chirp modulation transform and Fresnel transform. All of these have in common the calculation of quadratic phase factors of rapid oscillation. Such factors impose very restrictive sampling conditions; thus the methods are limited in their applicability.

In our particular case, our previous work has been dedicated to develop a Fourier-based algorithm for calculating light patterns in free space [3]. In [5], we presented a modified algorithm to calculate the cone of light from the exit of a convergent element to the distance of convergence. Three different methods were analysed and compared. We found that, in that case, the method based on angular spectrum propagation provides better results that the other two methods. The main

*Corresponding author. Email: david.mas@ua.es 
drawback of the process is that the Nyquist sampling limit requires an extremely large number of samples when high-power elements are studied.

Recently, Hennelly and Sheridan [6] have analysed the sampling requirements for any canonical transformation in optics. The study has been performed by considering geometrical transformations that any optical system introduces into the Wigner distribution function (WDF) of the signal. The paper states that, although the number of samples in both input and output spaces can be small, intermediate steps in the calculation algorithm may increase sampling requirements. In order to avoid interpolation and decimation at different stages of the algorithm, usually it is preferable to oversample input and output domains and to maintain the number of samples throughout the calculations. This may impose serious restrictions on the applicability of the algorithm in fast real-time processes.

One of the applications of these algorithms, also proposed by the present authors [8], is the analysis of the optical properties of the eye. The cornea and the crystalline lens are biological tissues which are not comparable with classical optical systems, in terms of image quality. Thus, image formation in the retina must be analysed by the conjunction of several factors, such as the distance of convergence, point spread function (PSF), modulation transfer function, Strehl ratio, symmetry of the peaks, and aberration coefficients [9]. In our particular case, we analyse the threedimensional PSF distribution, that is the whole light cone from the last optical surface of the system up to the distance of convergence. We would like to remark that the analysis of the light cone not only is applicable to the study of the eye but also can be extended to almost any optical system. The method is robust and allows analysis of new applications of diffractive optical elements to vision enhancement. As we stated above, the presence of high-power elements such as the cornea or the crystalline lens imposes important restrictions on the number of samples needed for the calculation, impeding very fast implementations.

As happens in any signal transmission system, the human eye has a frequencylimited response. The cut-off frequency may impose a critical number of samples, and sampling above this limit may reduce the speed of the process with ineffective precision. On the other hand, reducing sampling may introduce aliasing effects at intermediate steps of the algorithms and may distort the output result.

In this communication we propose a transformation that allows one to subsample patterns without losing quality, just by applying convenient scale factors on the illuminating wavelength. Since we have centred our attention on the spectrum propagation method, we shall briefly review the algorithm in section 2 . In section 3 we shall discuss the numerical implementation of the subsampling method. Some applications are sketched in section 4. Finally, in section 5 we offer a conclusion.

\section{Fresnel patterns calculation through angular spectrum propagation}

Let us consider a light beam of wavelength $\lambda$ that exits a phase element, so that it converges to a positive distance $z_{\mathrm{c}}$. Let us consider an input signal $u_{0}(x)$ located at the origin distance $z=0$. This signal has been discretized in $N$ samples, so that the 
discrete propagated pattern, calculated through an angular spectrum propagation method provides

$$
\left(u_{z}\right)_{\mu} \propto \mathrm{DFT}^{-1}\left\{\exp \left(-i \pi \frac{\lambda z}{\left(\Delta x_{0}\right)^{2}} \tilde{m}^{2}\right) \operatorname{DFT}\left[u_{0}\left(\frac{m \Delta x_{0}}{N}\right) \exp \left(-i \pi \frac{m^{2}\left(\Delta x_{0}\right)^{2}}{\lambda N^{2}} \frac{1}{z_{\mathrm{c}}}\right)\right]\right\},
$$

where $m, \tilde{m}$ and $\mu$ are discrete indices in space, the Fourier domain and the Fresnel domain respectively, DFT is the discrete Fourier transform and $\Delta x_{0}$ is the physical size of the object support window. $\Delta x_{0}$ must not be confused with the size of the pupil aperture, which must usually be taken to be smaller [2].

As detailed in [5], calculating all light patterns from the object at $z=0$ up to the theoretical convergence distance at $z=z_{\mathrm{c}}$ needs correct sampling of both quadratic phase factors in equation (1). The inner factor takes into account convergence of the beam. The Nyquist criterion applied upon this factor gives the following requirement for the number of samples:

$$
N \geq \frac{\Delta x_{0}^{2}}{\lambda z_{\mathrm{c}}}
$$

The external phase factor takes into account the propagation kernel and it is correctly sampled for distances

$$
z \leq \frac{\Delta x_{0}^{2}}{\lambda N} \equiv z_{\mathrm{c}}
$$

Equation (2) imposes an excessive number of sample points for very short focusing distances. As an example, if we consider the human cornea, $z_{\mathrm{c}}=32 \mathrm{~mm}$ and, for $\lambda=633 \mathrm{~nm}$ and $\Delta x_{0}=6 \mathrm{~mm}$, correct sampling of the convergent phase factor needs $N \approx 1800$ samples, with a distance between samples of about $\delta x_{0}=\Delta x_{0} / N \approx 5 \lambda$. Two-dimensional evaluation of such a propagation cone will require almost $4 \times 10^{6}$ samples. Although this sampling guarantees that the surface is correctly described, if the refracting element is sufficiently regular, one can guess that we do not need $4 \times 10^{6}$ rays for describing a caustic or a propagation cone.

From another point of view, the problem can be analysed under a Wigner distribution perspective. The WDF of a complex optical amplitude distribution provides a graphical means of simultaneously representing the signal's spatial and frequency distributions [10]. The WDF of a signal $u(x)$ is defined as

$$
W_{u}(x, \xi)=\int u\left(x+\frac{x^{\prime}}{2}\right) u^{*}\left(x-\frac{x^{\prime}}{2}\right) \exp \left(-i 2 \pi x^{\prime} \xi\right) \mathrm{d} x^{\prime}
$$

where $\xi$ represents the spatial frequency. The properties of the WDF and its transformations under the effect of an optical transformation can be found in the [10] and will not be detailed here. In most practical problems, it is assumed that the object is bounded in both spatial and frequency domains. The spatial extent $\Delta x$ and the frequency extent $\Delta \xi$ are defined such that both the signal and its Fourier transform are negligible outside their respective limits [11]. Following this notation, 
the number of samples needed to describe the signal completely is

$$
N \geq \Delta x \Delta \xi
$$

which is just the expression for the Nyquist condition.

The algorithm described in equation (1) produces the following distortion on the final WDF:

$$
\begin{aligned}
& \text { object } W_{u_{0}}(x, \xi) \\
& \text { convergence } W_{u_{0}^{\mathrm{c}}}(x, \xi)=W_{u_{0}}\left(x, \xi+\frac{x}{\lambda z_{\mathrm{c}}}\right) \\
& \text { propagation } W_{u_{z}}(x, \xi)=W_{u_{0}}\left(x-\lambda z \xi, \xi+\frac{x}{\lambda z_{\mathrm{c}}}\right) .
\end{aligned}
$$

These transformations will, in general, increase the bounds in both the spatial and the frequency domains and, following equation (5), sampling requirements will also increase. Proper selection of the input calculation window $\Delta x_{0}$ and the number $N$ of samples guarantees correct sampling of the transformed pattern, within the limitations of the selected numerical method $[5,6]$. In equations (6), two shearing distortions must be taken into account. That due to the convergence factor imposes a severe distortion, which also means severe broadening of the frequency bounds. Thus, the number of samples must be fixed taking into account this distortion. On the other hand, propagation produces a small deformation for short propagation distances. Thus, once $N$ is fixed, the Nyquist requirement imposes a restriction on the range of $z$ where the algorithm is applicable [6].

Note that, in all this process, the wavelength $\lambda$ acts as a scale parameter which is independent of all sampling conditions. Increasing the wavelength will reduce the shearing in the frequency domain and, as a consequence of this, the sampling requirements, moreover, if we accept that the frequency limits of the signal are decreased, according to the cut-off frequency of the transmission system, an important reduction in $N$ can be accomplished without violating the Nyquist condition in equations (2) and (3).

\section{Subsampling Fresnel patterns}

Let us separate the extrinsic variables of our problem such as sampling points and illuminating wavelength, namely $N$ and $\lambda$, from the other two variables that are intrinsic to the optical system. Thus, the Nyquist condition in equation (2) is

$$
N \lambda \geq \frac{\Delta x_{0}^{2}}{z_{\mathrm{c}}}
$$

Let us consider a given $N$ and $\lambda$. Both parameters can be modified while the product remains constant. Since we are interested in reducing the sampling requirement, 
we define a factor $\kappa>1$ so that

$$
\begin{aligned}
N^{\prime} & =\frac{N}{\kappa}, \\
\lambda^{\prime} & =\kappa \lambda .
\end{aligned}
$$

This means that we reduce sampling requirements by scaling the wavelength, but the Nyquist condition remains unchanged. Following inequalities (2) and (3), no aliasing is introduced into any quadratic phase factors.

In analytical calculations, introduction of scaled factors can be interpreted as changes in dummy variables but, in discrete calculations, these changes may affect to the space-bandwidth product. Hence, changing $N$ to $N^{\prime}$ and maintaining $\Delta x_{0}$ unchanged result in decimation in the spatial domain or, equivalently, a low-pass filter:

$$
\Delta \xi=\frac{1}{\delta x_{0}} \Rightarrow \Delta \xi^{\prime}=\frac{1}{\delta x_{0}^{\prime}}=\frac{\Delta \xi}{\kappa} .
$$

The use of two DFTs in equation (1) provides the following relation between spatial, Fourier and Fresnel domains:

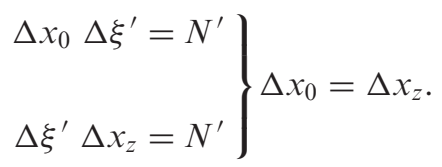

Hence, this subsampled Fresnel domain is just an apodized version of the original pattern.

Coming back to the Wigner representation, the change introduces the following transformation:

$$
\begin{aligned}
W_{u_{z}^{\prime} z}\left(x^{\prime}, \xi^{\prime}\right) & =W_{u_{0}}\left(x-\lambda^{\prime} z \xi^{\prime}, \xi^{\prime}+\frac{x}{\lambda^{\prime} z_{\mathrm{c}}}\right) \\
& =W_{u_{0}}\left(x-(\kappa \lambda) z \frac{\xi}{\kappa}, \frac{\xi}{\kappa}+\frac{x}{\kappa \lambda z_{\mathrm{c}}}\right) \\
& =W_{u_{0}}\left[x-\lambda z \xi, \frac{1}{\kappa}\left(\xi+\frac{x}{\lambda z_{\mathrm{c}}}\right)\right] \\
& \equiv W_{u_{z}}\left(x, \frac{\xi}{\kappa}\right),
\end{aligned}
$$

which can be interpreted as an anamorphic scaling in the frequency domain.

In figures $1(a)$ and $(b)$, we show the effect of resampling on the amplitude and phase on a test function. We have selected a Gaussian function centred on the optical axis with a total size $\Delta x_{0}=6 \mathrm{~mm}$ and a standard deviation $\sigma=1.06 \mathrm{~mm}$, we have set the convergence distance at $z_{\mathrm{c}}=64 \mathrm{~mm}$, and we have propagated the pattern following equation (1) up to $z=30 \mathrm{~mm}$. The integral has been sampled with $N=1024$ and the wavelength has been fixed at $\lambda=633 \mathrm{~nm}$. We have represented the subsampled pattern in front of the original pattern. Note that no important distortion is introduced although we have subsampled the integral by a factor $\kappa=8$. 


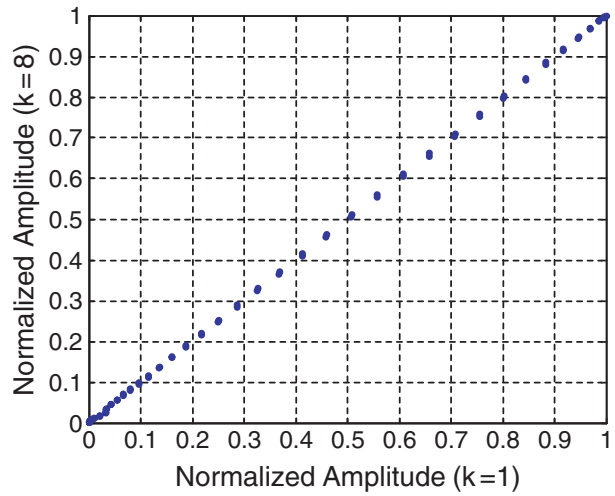

(a)

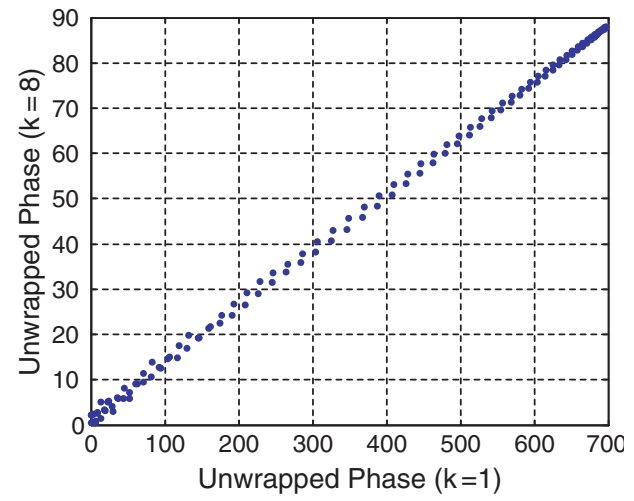

(b)

Figure 1. Comparative results for a Gaussian object with $\Delta x_{0}=6 \mathrm{~mm}$ and $\sigma=1.06 \mathrm{~mm}$, illuminated by a beam with $\lambda=633 \mathrm{~nm}$ which converges to $z_{\mathrm{c}}=64 \mathrm{~mm}:$ (a) comparison between amplitudes for a propagated pattern at a distance $z=30 \mathrm{~mm}$, with $\kappa=1$ and $\kappa=8$, corresponding to $N=1024$ and $N=128$ samples respectively; $(b)$ comparison between phase arguments for $\kappa=1$ and $\kappa=8$, unwrapped to $2 \pi$. (The colour version of this figure is included in the online version of the journal.)

In both cases we obtain a linear correspondence between the original pattern and the subsampled pattern. Note that the phases of both patterns in figure $1(b)$ are related by a proportionality factor equal to the parameter $\kappa$.

\section{Applications of the method}

As we stated before, one of the motivations of this work is the study of light propagation through the human eye $[12,13]$. Analysis of image formation in the human eye consists in studying not only the convergence of the beam but also the whole structure of the wavefront through aberration polynomials. The changes that we have proposed in sampling and $\lambda$ may introduce some decoupling in the different terms of the polynomial development.

Let us consider that, apart from a convergence term we introduce into our system a generic aberration function. Thus, in equation (1), the convergent phase factor must be substituted by a transmittance factor of the form

$$
t\left(m \frac{\Delta x_{0}}{N}\right)=\exp \left[i \frac{2 \pi}{\lambda} W\left(m \frac{\Delta x_{0}}{N}\right)\right],
$$

which has been expressed in discrete form.

Let us write this function as a polynomial expansion in Cartesian variables. The generic form of one term of the expansion, in both continuous and discrete form, is

$$
\exp \left(i \frac{2 \pi}{\lambda} \beta_{n} x_{0}^{n}\right) \rightarrow \exp \left(i \frac{2 \pi}{\lambda} \beta_{n} m^{n} \frac{\Delta x_{0}^{n}}{N^{n}}\right)
$$


Following the Nyquist criterion, this factor is correctly sampled provided that the total number of samples fulfils

$$
N \geq \frac{n \beta_{n}\left(\Delta x_{0}\right)^{n}}{\lambda 2^{n-2}}
$$

Note that we can isolate the product of $N$ and $\lambda$, so that the change proposed in equation (8) does not affect the Nyquist limit.

As before, let us suppose that we calculate a pattern with modified parameters $N^{\prime}$ and $\lambda^{\prime}$. Bearing in mind equations (8), the discrete index may be transformed as $m^{\prime}=m / \kappa$, and as a result the exact phase factor that we are calculating is

$$
\exp \left(i \frac{2 \pi}{\lambda^{\prime}} \beta_{n} m^{\prime n} \frac{\Delta x_{0}^{n}}{N^{\prime n}}\right)=\exp \left(i \frac{2 \pi}{\lambda} \frac{\beta_{n}}{\kappa} m^{n} \frac{\Delta x_{0}^{n}}{N^{n}}\right) .
$$

Thus, the effect is equivalent to calculating the original polynomial development with scaled coefficients.

From equation (15) we can conclude that adequate subsampling and rescaling of the wavelength allow an important reduction in the number of samples needed for correct calculation of Fresnel patterns from the object to a convergence point. In the case of the human cornea, let us remember that $z_{\mathrm{c}}=32 \mathrm{~mm}$ and, for $\lambda=633 \mathrm{~nm}$ and $\Delta x_{0}=6 \mathrm{~mm}$, correct sampling needs $N \geq 1800$ samples, with a distance between samples of about $\delta x_{0}=\Delta x_{0} / N \approx 5 \lambda$. This means that the cut-off frequency induced by the method is about $\Delta \xi=300 \mathrm{~mm}^{-1}$ or, equivalently, about $\Delta \xi=168 \mathrm{c} \mathrm{deg}^{-1}$. Assuming that visual system can process up to $60 \mathrm{c} \mathrm{deg}^{-1}$ on average, we can assume a subsampling by a factor $\kappa=2$ without losing any relevant information that is visible for the eye. This reduction is quadratic in two-dimensional calculations, so that we can divide by 4 our requirements without introducing any aliasing in our system. Further reduction is possible if we assume a cut-off frequency below $60 \mathrm{cdeg}^{-1}$.

\section{Conclusion}

The proposed method allows subsampling Fresnel patterns without introducing aliasing. The reduction in the number of samples is achieved by rescaling $\lambda$ by the inverse factor, thus maintaining their product constant. This does not affect the Nyquist sampling condition but introduces an effective low-pass filter. As a consequence of this, the method is applicable when the cut-off frequency of the system is below the requirements of the propagation algorithm.

The problem has been analysed from the algorithmic point of view and under the Wigner charts description. This formalism allow better understanding of the transformations that numerical propagation algorithms introduce into the space-bandwidth product.

The final effect of this rescaling on the number of samples and the wavelength is the same as is introduced by rescaling the coefficients of the aberration polynomial. Resampling implies important savings in computer memory requirements and in 
computing time, thus allowing implementation of the algorithm in real-time processes.

\section{Acknowledgments}

This work has been supported by the Conselleria de Empresa, Universitat i Ciència of the Generalitat Valenciana, through project GV04A/578.

\section{References}

[1] J. García, D. Mas and R.G. Dorsch, Appl. Optics 357013 (1966).

[2] D. Mendlovic, Z. Zalevsky and N. Konforti, J. Mod. Optics 44407 (1997).

[3] D. Mas, J. Garcia, C. Ferreira, et al., Optics Commun. 164233 (1999).

[4] M.V. Papalexandris and D.C. Redding, J. Opt. Soc. Am. A 101763 (2000).

[5] D. Mas, J. Pérez, C. Hernández, et al., Optics Commun. 227245 (2003).

[6] B.M. Hennelly and J.T. Sheridan, J. Opt. Soc. Am. A 22917 (2005).

[7] B.M. Hennelly and J.T. Sheridan, J. Opt. Soc. Am. A 22928 (2005).

[8] C. Illueca, D. Mas, J. Pérez, et al., J. Mod. Optics 48811 (2001).

[9] D. Mas, J. Pérez, C. Vázquez, et al., J. Mod. Optics 501335 (2003).

[10] A.W. Lohmann, J. Opt. Soc. Am. A 102181 (1993).

[11] A.W. Lohmann, R.G. Dorsch, D. Mendlovic, et al., J. Opt. Soc. Am. A 13470 (1996).

[12] J. Pérez, D. Mas, J.J. Miret, C. Vázquez, et al., Optik 11649 (2005).

[13] J. Pérez, D. Mas, C. Illueca, et al., J. Mod. Optics 521161 (2005). 